\title{
SEGUIMENTO DOS ENFERMEIROS EGRESSOS DOS CURSOS DE ESPECIALIZAÇÃO EM ENFERMAGEM EM CUIDADOS INTENSIVOS
}

\author{
Verlaine Andrade* \\ Kátia Grillo Padilha** \\ Miako Kimura**
}

\begin{abstract}
ANDRADE, V.; PADILHA, K.G.; KIMURA, M. Seguimento dos enfermeiros egressos dos cursos de especialização em enfermagem em cuidados intensivos. Rev.latino-am.enfermagem, Ribeirão Preto, v. 6, n. 3, p. 23-31, julho 1998.
\end{abstract}

O presente estudo busca analisar a contribuição trazida pelos Cursos de Especialização em Cuidados Intensivos realizados na Escola de Enfermagem da USP, cujos objetivos foram: 1) caracterizar a trajetória dos enfermeiros quanto à continuidade do trabalho em UTI; 2) avaliar a contribuição oferecida pelo curso. A população foi composta por 38 enfermeiros egressos, sendo que, destes, 60,5\% não mais atuavam na UTI, devido ao fato de assumirem outro cargo na instituição e apresentarem problemas pessoais diversos. Quanto à contribuição oferecida pelo curso, a principal avaliação recaiu na aquisição de conhecimentos $(91,7 \%)$. No entanto, $74,2 \%$ dos enfermeiros afirmaram que o curso não contribuiu para o aumento salarial, o que não os têm impedido de investir no seu aperfeiçoamento profissional.

Unitermos: enfermagem em terapia intensiva, ensino em UTI

\section{INTRODUÇÃO}

A implementação de Cursos de Especialização em áreas diversas na Enfermagem tem se mostrado cada vez mais necessária e emergente.

Tendo por objetivo fundamental atender a uma demanda específica do mercado de trabalho, aprofundando e completando conhecimentos, habilidades e atitudes necessárias ao domínio de funções bem definidas no seu perfil técnico-científico, a especialização em cuidados intensivos se impõe, sobretudo pelo avanço progressivo e rápido dos novos conhecimentos na área, bem como pelo aparato tecnológico presente nas Unidades de Terapia Intensiva.

Segundo KOIZUMI et al. (1985), ao se propor um Curso de Especialização é necessário em primeiro lugar, avaliar-se a real necessidade do mercado de trabalho, assim como a demanda existente, ambas analisadas quanto às suas características e distribuição. Para essas autoras, o diagnóstico resultante desta avaliação, por sua vez, constituirá a base para se programar um conjunto coerente de atividades, capaz de promover a completa adaptação de um graduado às suas funções específicas, no caso, o atendimento ao paciente grave em UTI.

Partindo-se dos pressupostos referidos, foram implementados na Escola de Enfermagem da USP, desde 1982, Cursos de Especialização em Enfermagem em Cuidados Intensivos, inicialmente com periodicidade anual e a partir de 1988, bienalmente, tendo por base normativa as Resoluções $n^{\circ}$ 14/77 do Conselho Federal de Educação (BRASIL, 1979) e no 1709/79 da Universidade de São Paulo (USP, 1979).

Após a realização de nove Cursos na citada modalidade, sentiu-se a necessidade de, frente à nova determinação do Conselho Federal de Enfermagem*** que versa sobre as especializações, analisar até que ponto os cursos já oferecidos têm contribuído para o desenvolvimento das atividades profissionais dos enfermeiros na UTI.

Além da avaliação desse aspecto, acredita-se que o "follow-up" desses profissionais poderá ainda trazer importantes indicativos sobre o investimento feito pelo enfermeiro na continuidade da sua formação profissional, através da realização de outros cursos, como também no desenvolvimento de novos projetos de pesquisa, além

\footnotetext{
* Estudante do Curso de Graduação em Enfermagem e Obstetrícia da Escola de Enfermagem da Universidade de São Paulo

** Professor Doutor do Dept ${ }^{\circ}$ de Enfermagem Médico-Cirúrgica da Escola de Enfermagem da Universidade de São Paulo

*** Resolução COFEN - 173. Rio de Janeiro, jun 1994
} 
daquele apresentado no transcorrer do Curso de Especialização.

Considerando-se ainda que a abrangência do ensino na Universidade deve contemplar além da graduação, a pós-graduação lato e stricto sensu, de forma integrada, julgamos que o seguimento dos enfermeiros egressos dos cursos de especialização poderia, inclusive, subsidiar futuras articulações entre as diferentes modalidades de ensino na área de cuidados intensivos.

Diante do exposto, propõe-se a realização do presente estudo cujos objetivos são:

- Caracterizar a trajetória dos enfermeiros após a realização do Curso de Especialização em Enfermagem em Cuidados Intensivos quanto à continuidade do trabalho em UTI, ao prosseguimento dado à formação profissional, ao seguimento dado à pesquisa realizada no decorrer do curso e ao desenvolvimento de novos projetos de pesquisa.

- Avaliar o grau de contribuição do curso atribuído pelos enfermeiros egressos dos Curso de Especialização em Cuidados Intensivos para a sua prática profissional.

\section{METODOLOGIA}

\subsection{População}

Dos 134 enfermeiros que frequentaram os Cursos de Especialização em Enfermagem em Cuidados Intensivos da Escola de Enfermagem da Universidade de São Paulo (EEUSP), conseguiu-se a localização de 89 deles, o equivalente a $66,4 \%$ do total de egressos. Destes, obteve-se o retorno de 45 instrumentos $(50 \%)$ dos quais, 7 retornaram ao remetente devido a não localização dos destinatários.

Assim, a população do presente estudo foi constituída por 38 enfermeiros.

\subsection{Coleta de Dados}

A coleta de dados foi realizada durante 3 meses, período que se considerou necessário para a localização e/ou confirmação do endereço dos enfermeiros, bem como para o envio e retorno dos questionários.

\subsubsection{Instrumento}

Para a coleta de dados foi elaborada uma carta (Anexo 1) onde se solicitou a participação do enfermeiro na pesquisa. $\mathrm{O}$ instrumento específico utilizado para a coleta foi um questionário (Anexo 2) elaborado com perguntas abertas e fechadas, composto de duas partes. A primeira refere-se a dados gerais de caracterização da população quanto a idade, sexo, tempo de formado e tempo de trabalho em UTI. A segunda está diretamente relacionada aos objetivos da pesquisa, ou seja: continuidade ou não do trabalho em UTI e tempo de atuação nessa unidade após o término do Curso de Especialização; tipo de atividade que exerce atualmente; outros cursos realizados; contribuição do Curso para as atividades profissionais e desenvolvimento de pesquisas posteriormente ao curso. Reservou-se também um espaço para comentários e sugestões.

\subsubsection{Operacionalização da Coleta de Dados}

A listagem dos enfermeiros matriculados nos Cursos de Especialização em Cuidados Intensivos, bem como a relação dos respectivos telefones e endereços foi solicitada ao Serviço de Cultura e Extensão da EEUSP, seção responsável pelos Cursos de Especialização, sensu latu, de curta duração (360 horas).

A busca dos enfermeiros cujos endereços não foram obtidos por essa via, deu-se através de lista fornecida pela Associação Brasileira de Enfermagem Seção São Paulo.

Uma vez obtidos os endereços, os instrumentos de coleta de dados foram encaminhados via correio, com envelope selado para o envio das respostas.

\subsection{Tratamento estatístico}

Os dados foram analisados por meio de números absolutos e percentuais.

\section{RESULTADOS E DISCUSSÃO}

\subsection{Caracterização da população}

A população do estudo foi constituída por maioria feminina, ou seja, $89,6 \%$, enquanto que a masculina representou apenas 5,2\%. Ressalta-se que 2 enfermeiros não responderam a essa questão.

No que se refere a idade, verificou-se que a idade mínima foi de 24 anos e a máxima de 45 anos, com média equivalente a 34,5 anos. A distribuição por faixa etária permitiu constatar que 27 enfermeiros $(71,0 \%)$ encontravam-se na faixa compreendida entre 30 e 40 anos, enquanto que $5(13,2 \%)$ e $4(10,5 \%)$ tinham entre 20 e 30 anos e 40 e 50 anos, respectivamente. Dois enfermeiros $(5,3 \%)$ não forneceram essa informação. 
Tabela 1 - Distribuição dos enfermeiros segundo o tempo de formado e o tempo de trabalho em UTI. São Paulo, 1995

\begin{tabular}{|c|c|c|c|c|c|c|c|c|}
\hline \multirow{2}{*}{$\begin{array}{r}\text { Tempo de trabalho } \\
\text { em UTI } \\
\text { Tempo de formado }\end{array}$} & \multicolumn{2}{|c|}{$1 \leftrightarrow 5$} & \multicolumn{2}{|c|}{$6 \leftrightarrow 10$} & \multicolumn{2}{|c|}{$11 \leftrightarrow 15$} & \multicolumn{2}{|c|}{ TOTAL } \\
\hline & $\mathrm{n}^{\circ}$ & $\%$ & $\mathrm{n}^{\circ}$ & $\%$ & $\mathrm{n}^{\circ}$ & $\%$ & $\mathrm{n}^{\circ}$ & $\%$ \\
\hline $1 \leftrightarrow 5$ & 3 & 7,8 & - & - & - & - & 3 & 7,8 \\
\hline $6 \leftrightarrow 10$ & 5 & 13,1 & 6 & 15,8 & - & - & 11 & 28,9 \\
\hline $11 \leftrightarrow 15$ & 7 & 18,4 & 9 & 23,7 & 3 & 7,8 & 19 & 50,0 \\
\hline $16 \leftrightarrow 20$ & - & - & 2 & 5,2 & 2 & 5,2 & 4 & 10,3 \\
\hline Branco & - & - & - & - & - & - & 1 & 3,0 \\
\hline TOTAL & 15 & 39,3 & 17 & 44,7 & 5 & 13,0 & 38 & 100,0 \\
\hline
\end{tabular}

Observa-se pelos dados da Tabela 1 que 19 enfermeiros $(50,0 \%)$ estavam formados entre 11 e 15 anos e que 17 enfermeiros $(44,7 \%)$ referiram ter atuado na UTI por um período compreendido entre 6 e 10 anos. Pelos resultados demonstrados, cabe destacar que ao somarmos àqueles 17 enfermeiros, outros $5(13,0 \%)$ que atuaram na UTI entre 11 e 15 anos, constata-se que 22 enfermeiros $(57,7 \%)$ têm um tempo longo de trabalho em UTI, apesar de todas as dificuldades apontadas para a permanência dos trabalhadores de enfermagem nessa área, em função do tipo estressante de trabalho nela desenvolvido.

Dados semelhantes foram encontrados no estudo de SPÍNDOLA et al (1994), no qual se verificou que $69,2 \%$ da população estava formada há mais de 9 anos e que $38,6 \%$ atuavam na UTI por mais de 10 anos. A faixa etária prevalente (30-34 anos) é sugestiva de profissionais adultos jovens, assim como no presente estudo, porém com experiência na área, pressupondo uma força de trabalho compatível à complexidade e ao trabalho desenvolvido na unidade.

\subsection{Caracterização do "follow-up" após o Curso de Especialização em Enfermagem em Cuidados Intensivos}

Referente à continuidade do trabalho do enfermeiro em UTI após a realização do curso, constatouse que apenas $14(36,8 \%)$ referiram ainda permanecer na UTI, enquanto que a maioria, ou seja, $23(60,5 \%)$ não mais atuava nessa área. Um respondente não forneceu essa informação.

Analisando-se o período de tempo que o enfermeiro permaneceu na UTI, após a realização do curso, pôde-se verificar que o menor período foi de 8 meses e o maior, 11 anos, distribuídos conforme dos dados da Tabela 2.
Tabela 2 - Distribuição dos enfermeiros segundo o tempo de permanência na UTI após a realização do curso de especialização. São Paulo, 1995

\begin{tabular}{lcc}
\hline $\begin{array}{l}\text { Tempo de Trabalho após o } \\
\text { curso (anos) }\end{array}$ & $\mathrm{N}^{\circ}$ de respostas & $\%$ \\
\hline $1 \leq \mid-3$ & 11 & 29,0 \\
$3 \mid-5$ & 12 & 31,6 \\
$5 \mid-7$ & 5 & 13,2 \\
$7|-| \geq 9$ & 8 & 21,0 \\
Branco & 2 & 5,2 \\
\hline TOTAL & 38 & 100,0 \\
\hline
\end{tabular}

Pelos dados da Tabela 2, verifica-se que 12 enfermeiros $(31,6 \%)$ permaneceram na UTI por um período de 3 a 5 anos, seguido por $11(29,0 \%)$ que ali atuaram por até 3 anos. Buscando averiguar a permanência por período superior a 5 anos, foi interessante observar que 13 enfermeiros (34,2\%) trabalharam na UTI por mais de 5 anos, após a realização do curso de especialização. Os dados obtidos nos levam a pensar que, embora $60,5 \%$ dos enfermeiros não mais se encontravam na UTI, o tempo nela permanecido, após o curso, pode ser considerado como suficiente para a aplicação dos conhecimentos adquiridos, no que se refere às especificidades do cuidado ao paciente crítico.

Considerando, no entanto, que grande contingente de enfermeiros se afastaram da UTI, procurou-se investigar os motivos que os levaram a tal decisão. Tais resultados encontram-se apresentados na Tabela 3.

Tabela 3 - Justificativas apresentadas pelos enfermeiros para saírem da UTI. São Paulo, 1995

\begin{tabular}{lcc}
\hline Justificativas & $\mathrm{N}^{\circ}$ & $\%$ \\
\hline Problemas pessoais diversos & 6 & 30,0 \\
Ocupação de outro cargo na & 6 & 30,0 \\
institução & 4 & 20,0 \\
Mudaça de área de atuação & 2 & 10,0 \\
Mudança de emprego & 2 & 10,0 \\
Outros* & & \\
\hline TOTAL & 20 & 100,0 \\
\hline
\end{tabular}

* Problemas administrativos; ausência de enfermeira exclusiva na UTI 
Do total de 23 enfermeiros que deixaram de atuar em UTI, 3 não apresentaram justificativas para a sua saída, perfazendo, desse modo uma justificativa por enfermeiro.

Das respostas apresentadas, verifica-se que igual percentagem, ou seja, $30,0 \%$ dos motivos recaíram sobre problemas pessoais diversos e ocupação de outro cargo na instituição, seguidos por mudança de área de atuação $(20,0 \%)$.

Dentre os motivos relacionados a problemas pessoais, o cansaço, o desânimo, o stress e os problemas relacionados ao rodízio de horário foram os mais referidos.

SPÍNDOLA et al. (1994) e BARRY (1986) classificam a UTI como uma unidade geradora de estresse, destacando como fatores desencadeantes mais comuns, a sobrecarga de trabalho, a convivência com situação de vida e morte, o risco de vida, o ambiente de crise, os eventuais conflitos com a administração e a má utilização dos recursos médicos disponíveis. BARRY (1986) acrescenta ainda, a morte do paciente, problemas diversos de comunicação e o grande número de decisões imediatas a serem tomadas pelos profissionais. Autores como TAKAHASHI (1985); TAKAHASHI (1991); PADILHA (1994); CLARK et al. (1994) corroboram tais resultados.

Quanto à ocupação de outro cargo na instituição constatou-se que as justificativas foram relacionadas principalmente ao fato de assumirem cargos administrativos e de ensino, destacando-se o desenvolvimento de atividades diretamente relacionadas à educação continuada.

Diante dessas justificativas, pode-se inferir que os enfermeiros, por terem atuado em UTI e terem investido na sua formação acadêmica, sejam profissionais considerados mais experientes e habilitados, o que os leva a serem remanejados para o desenvolvimento de atividades de maior abrangência na instituição. Esse fato atrelado à possibilidade de trabalhar em horário fixo no período diurno, bem como atuar numa área menos estressante acabam por determinar a saída do enfermeiro da UTI.

Referente às atividades exercidas pelos enfermeiros atualmente, constatou-se que nenhum deles abandonou totalmente a sua opção profissional. Apenas um mencionou ter uma pequena empresa, porém continua atuando como enfermeiro-docente num curso de nível médio.

Ainda focalizando as atividades profissionais atuais, observou-se que dos 23 enfermeiros que não mais continuam na UTI, 6 exercem atividade docente (nível auxiliar, técnico ou superior), 6 atuam em unidades hospitalares diversas, enquanto que 3 mudaram totalmente de área, passando a trabalhar em Saúde
Pública. Os demais enfermeiros, em número de 8 , referiram desenvolver atividades relacionadas à educação continuada e à prestação de serviços, dentre outros.

Por outro lado, dos 14 enfermeiros que permaneceram na UTI, 6 continuam atuando como assistenciais, 3 desenvolvem atividade administrativa e 4 desempenham atividade docente ou docente-assistencial na área de cuidados intensivos. Uma justificativa foi deixada sem resposta.

Analisando-se os resultados obtidos, pode-se dizer que, provavelmente, os enfermeiros que deram seguimento a sua atuação na UTI foram os que mais aproveitaram o curso de especialização no que se refere à aplicabilidade específica do conteúdo teórico-prático adquirido. É de se supor também que os demais profissionais, apesar de ocuparem diferentes cargos e funções, tenham se beneficiado, haja visto a continuidade do trabalho em outras unidades hospitalares (médicocirúrgicas) bem como na docência. Obviamente a fundamentação teórico-prática adquirida qualificam a assistência e o ensino nessas outras atividades.

Considerando-se o investimento feito para a continuidade da formação profissional, 31 enfermeiros $(81,6 \%)$ realizaram outros cursos após a especialização em UTI totalizando 43, o que perfaz uma média de 1,4 curso/enfermeiro. Destes, $9(20,9 \%)$ referem-se à pósgraduação sensu strictu, mestrado (7) e doutorado (2). No entanto, o maior número de cursos são do tipo sensu latu e estão distribuídos entre especialização (16), atualização (16) e licenciatura (2) somando 34 ou 79,1\%. É importante acrescentar que, dos 16 cursos de especialização realizados, 10 estão relacionados à área administrativa, o que é, de certa forma, condizente com as atividades desenvolvidas pelos enfermeiros de forma geral, seja na UTI ou fora dela.

Salienta-se que dos 14 enfermeiros que continuaram na UTI, 8 fizeram pós-graduação sensu latu, 3 fizeram sensu strictu e apenas 3 não fizeram nenhum outro curso. Numa análise geral, os dados permitem dizer que os profissionais que fizeram o curso de especialização em cuidados intensivos têm um perfil que valoriza a continuidade da própria educação, visando a um melhor desempenho profissional, independentemente de continuarem na UTI ou não.

Sendo um dos objetivos do curso o desenvolvimento de um trabalho de iniciação científica, procurou-se investigar o encaminhamento dado, posteriormente ao curso, à pesquisa realizada.

Dos 38 enfermeiros respondentes, 2 informaram não ter realizado tal atividade, por não ser exigência no primeiro ano de realização do curso. Os resultados obtidos demonstraram que 25 enfermeiros $(65,8 \%)$ implementaram os dados da pesquisa na sua prática cotidiana, $13(34,2 \%)$ o apresentaram em evento científico 
(Congressos, Jornadas, etc), sendo que apenas 4 (10,5\%) chegaram a publicá-lo.

Embora seja bastante positivo o fato da maioria dos enfermeiros implementarem na prática os resultados obtidos com a investigação realizada, nota-se que a sua divulgação não teve o mesmo encaminhamento, apesar de $34,2 \%$ dos enfermeiros o apresentarem em algum tipo de evento. Lamentável também foi verificar que apenas 10,5\% dos enfermeiros efetivaram a sua publicação.

Esses resultados, frente aos obtidos por KOIZUMI et al. (1986); PADILHA et al. (1995) em estudo sobre a produção científica em enfermagem em cuidados intensivos, onde os autores encontraram uma produção muito restrita de trabalhos nesta área, são no mínimo, preocupantes. Como formar um corpo de conhecimentos específicos sem uma disseminação mais abrangente das pesquisas desenvolvidas?

Investigando as justificativas apresentadas para a não publicação do estudo, percebeu-se que a maior porcentagem de respostas $(15-41,6 \%)$ foi relacionada ao desinteresse dos autores, seguida pelas dificuldades inerentes ao próprio grupo $(9-25 \%)$, principalmente à falta de tempo e à dispersão dos colegas. Cabe ressaltar que esses mesmos motivos levaram a não apresentação do estudo em eventos científicos.

Diante desses dados, um questionamento que emerge refere-se ao valor que é dado pelos enfermeiros para o desenvolvimento de pesquisa, sua aplicação prática e divulgação dos resultados.
Nesse sentido, as dificuldades inerentes ao desenvolvimento e publicação de trabalhos científicos de enfermagem, no Brasil, são conhecidas e têm sido apontadas por vários autores (ALMEIDA, 1995; SOUSA \& GUTIERREZ, 1990; FERNANDES \& SILVA,1995; SILVA et al., 1991; ANGERAMI, 1993). No entanto, todos são unânimes em afirmar que a pesquisa é um desafio a ser superado pelos enfermeiros, não se justificando posturas de desinteresse e desânimo, conforme observado em $41,6 \%$ das justificativas apresentadas para a não publicação das pesquisas realizadas.

Ainda relacionado ao desenvolvimento do trabalho científico, foi de interesse identificar se outros estudos foram desenvolvidos no decorrer da vida profissional, após o curso de especialização.

Constatou-se que 22 enfermeiros (57,9\%) relataram a não participação em outras pesquisas, enquanto que $16(42,1 \%)$ referiram ter desenvolvido novos estudos, num total de 22 , verificando-se mais do que um trabalho realizado por enfermeiro.

Nesse sentido, cabe ressaltar a vinculação dos enfermeiros a cursos de pós-graduação sento strictu, local onde há maior exigência quanto à produção científica, $\mathrm{o}$ que provavelmente contribuiu para que $42,1 \%$ dos enfermeiros desenvolvessem novos estudos.

Procurando-se investigar a avaliação feita pelos enfermeiros no que se refere ao conteúdo específico do curso, obteve-se os resultados apresentados a seguir.

Tabela 4 - Contribuição do curso atribuída pelos enfermeiros, segundo itens específicos. São Paulo, 1995

\begin{tabular}{l|cccccc|c}
\hline \multicolumn{1}{c|}{ Avaliação } & \multicolumn{2}{c}{ Muita } & \multicolumn{2}{c}{ Pequena } & \multicolumn{2}{c}{ Nenhuma } & \multicolumn{2}{c}{ Total } \\
Itens & $\mathrm{n}^{\circ}$ & $\%$ & $\mathrm{n}^{\circ}$ & $\%$ & $\mathrm{n}^{\circ}$ & $\%$ & $\mathrm{n}^{\circ}$ \\
\hline Conhecimento técnico-científico & 33 & 91,7 & 3 & 8,3 & - & - & 36 \\
Desempenho da assistencia & 32 & 88,9 & 3 & 8,3 & 1 & 2,8 & 36 \\
Relacion. com equipe de enfermagem & 23 & 63,9 & 10 & 27,8 & 3 & 8,3 & 36 \\
Relacion. com equipe multidisciplinar & 22 & 61,1 & 13 & 36,1 & 1 & 2,8 & 36 \\
Relacionamento com paciente e familia & 20 & 55,5 & 14 & 38,9 & 2 & 5,6 & 36 \\
Desevolvimento de pesquisas & 14 & 38,9 & 14 & 38,9 & 8 & 22,8 & 36 \\
Ascensão na carreira & 13 & 36,1 & 7 & 19,5 & 16 & 44,4 & 36 \\
Aumento salarial & 1 & 2,9 & 8 & 22,9 & 26 & 74,2 & $35 *$ \\
\hline
\end{tabular}

* Uma resposta em branco

Pelos dados da Tabela 4, observa-se que do total de 38 enfermeiros, 36 qualificaram os itens apresentados segundo o grau de contribuição, à exceção do tópico "aumento salarial".

Quanto à especificação dos itens para os quais a contribuição foi de muito valor, verifica-se que foram relacionados principalmente à aquisição de conhecimento técnico-científico (33-91,7\%) e melhor desempenho da assistência ao paciente (32 - 88,9\%), fato bastante positivo, uma vez que estes constituem os principais objetivos propostos pelo curso. Além desses, houve também a referência de muita contribuição, embora em menor frequência, para o item relacionamento com a equipe de enfermagem ( $23-63,9 \%)$, com a equipe multidisciplinar (22-61,1\%) e com paciente e família (20 - 55,5\%). Tais avaliações são muito relevantes quando se considera a assistência global ao paciente crítico, numa unidade fechada.

Em relação à pequena contribuição trazida pelo curso, nota-se que os itens desenvolvimento de pesquisa 
e relacionamento com paciente-família foram os mais citados (14 respostas ou 38,9\%), seguidos por relacionamento com a equipe multidisciplinar $(13-36,1 \%)$ e de enfermagem $(10-27,8 \%)$. Tais resultados vêm demonstrar que não obstante à contribuição trazida pelo curso, ênfase deve ser dada quer no conteúdo, quer nas estratégias de desenvolvimento daqueles aspectos, tentando preencher as lacunas existentes.

É interessante notar, por outro lado, que o curso não trouxe nenhuma contribuição para o aumento salarial dos enfermeiros (26 respostas ou 74,2\%), dados lamentáveis, que retratam a desvalorização da educação continuada, que deveria ser incentivada, estimulada e até mesmo proporcionada pela instituição.

Denota-se que a busca do curso de especialização parte quase que exclusivamente da motivação individual do enfermeiro, que procura maior capacitação, porém que sabe, à priori, que as chances de retorno financeiro efetivo são quase que inexistentes e as de ascensão à carreira bastante limitadas. Acresce-se ainda, como outro agravante, a não facilitação de horários de trabalho mais compatíveis com o curso, aspecto que facilitaria o rendimento do aluno e melhoraria, inclusive, sua qualidade de vida.

Em síntese, pode-se dizer que os resultados deste estudo forneceram importantes subsídios para a análise geral do curso, tanto no que se refere ao conteúdo específico, quanto no encaminhamento das pesquisas propostas durante o seu desenvolvimento. Permitiu ainda constatar a sua importância na formação dos enfermeiros intensivistas, justificando, portanto, a sua manutenção e aperfeiçoamento.

\section{CONCLUSÕES}

A análise dos resultados obtidos no estudo permitiram as seguintes conclusões:

- No que se refere à continuidade do trabalho em UTI, após a realização do Curso de Especialização, observouse que a maioria dos enfermeiros $(60,5 \%)$ não mais atuava em terapia intensiva, enquanto que $36,8 \%$ permaneciam desenvolvendo suas atividades profissionais nessa unidade. As principais justificativas apontadas para a saída da UTI foram problemas pessoais diversos e ocupação de outro cargo na instituição (30,0\% para cada um).

- A maioria dos enfermeiros egressos, ou seja, 31 (81,6\%) deu continuidade a sua formação profissional através da participação em outros 43 cursos (média de 1,4 curso/ enfermeiro) dos quais, 9 foram de pós-graduação sensu strictu (mestrado e doutorado) e 34 sensu latu (especialização e atualização).

- Quanto ao seguimento dado a pesquisa realizada no decorrer do curso, $65,8 \%$ dos enfermeiros implementaram os resultados na prática profissional, $34,2 \%$ apresentaram o trabalho em evento científico, enquanto que apenas $10,5 \%$ chegaram a publicá-lo. A maioria dos enfermeiros $(57,9 \%)$ não desenvolveu nenhum outro projeto de pesquisa após o curso de especialização em cuidados intensivos.

- Com relação à contribuição trazida pelo curso, "muita contribuição" foi atribuída pela maioria dos enfermeiros aos itens aquisição de conhecimento técnico-científico $(91,7 \%)$ e desempenho da assistência ao paciente (88,9\%). "Pequena contribuição" foi citada, em função principalmente do item aumento salarial $(74,2 \%)$.

\section{FOLLOWING UP NURSES THAT FINISHED THE SPECIALIZATION COURSE ON NURSING IN INTENSIVE CARE}

The purpose of this study was to analyse the contribution of the Extension Courses in the Intensive Care held at the Nursing School from USP, with the aim to: 1) Follow up nurses in their work at the ICU. 2) Evaluate the contribution offered by the course. The population was formed by 38 nurses, considering that 60,5\% didn't work in the ICU anymore mainly because they assumed another function in the institution and had several private problems. Regarding the contribution offered by the course, the main evaluation was the acquirement of knowledge $(91,7 \%)$. However, 74,2\% of the nurses declared the course didn't contribute to the increase of their wage, what do not impair them from investing in their professional improvement.

KEY WORDS: intensive care nursing, extension course

\section{SEGUIMIENTO DE LOS ENFERMEROS EGRESOS DE LOS CURSOS DE ESPECIALIZACIÓN EN ENFERMERÍA EN CUIDADOS INTENSIVOS}

Este estudio busca analizar la contribución de los Cursos de Especialización en Cuidados Intensivos ofrecidos por la Escuela de Enfermería de la Universidad de São Paulo. Los objetivos fueron: 1) caracterizar la trayectoria de los enfermeros en 
cuanto a la continuidad del trabajo en Unidade de Tratamiento Intensivo (UTI); 2) evaluar la contribución ofrecida por el Curso. La población fue de 38 enfermeros egresados de los Cursos, el 60,5\% no estaban trabajando en UTI por estar ejerciendo otro cargo y también por problemas personales. En cuanto a la contribución ofrecida por el Curso, el principal resultado de la evaluación apunta para la aquisición de conocimientos (91,7\%), el 74,2\% de los enfermeros dijeron que el Curso no contribuyó para la mejoría de la remuneración; sin embargo, eso no constituyó motivo para que esos enfermeros dejaran de invertir en el progreso profesional.

TÉRMINOS CLAVES: enfermagem em terapia intensiva, ensino em UTI

\section{ANEXO 1}

São Paulo, 13 de junho de 1995.

Prezado colega,

Vimos mais uma vez solicitar a sua valiosa colaboração no preenchimento do questionário anexo que se destina à obtenção de dados que nos possibilitam o acompanhamento dos ex-alunos dos Cursos de Especialização em Enfermagem em Cuidados Intensivos, minsitrados pela Escola de Enfermagem da USP.

Esses dados são, para nós, de particular interesse uma vez que estamos em vias de organizar o X Curso de Especialização em Enfermagem em Cuidados Intensivos para o ano de 1995, momento oportuno para visualizarmos a contribuição do mesmo para os enfermeiros de UTI.

Ressaltamos que será garantido o anonimato das informações fornecidas.

Contando com a sua colaboração, antecipadamente agradecemos, colocando-nos à disposição para os esclarecimentos que se fizerem necessários, pelo telefone 852.8922 (Ramal 414).

Kátia Grillo Padilha
Docente do Curso de Especialização em
Enfermagem em Cuidados Intensivos

Obs: Solicitamos a devolução do questionário, preenchido ou não, no envelope selado o mais rápido possível.

\section{ANEXO 2}

\section{QUESTIONÁRIO}

$\mathrm{N}^{\mathrm{o}}$

I - Dados gerais:

Idade:

Tempo de formado:

Tempo de trabalho em UTI:

II - Dados específicos:

1- Continua trabalhando em UTI?

( ) $\operatorname{Sim}$

( ) Não. Por que motivo saiu? 
Por quanto tempo atuou em UTI após o curso de especialização?

anos meses

Que atividade exerce atualmente:

*na enfermagem:

* fora da enfermagem:

2- Outros cursos realizados depois da especialização em cuidados intensivos:

( ) Não

( ) Sim ( ) Doutorado

( ) Mestrado

( ) Atualização

( ) Especialização. Especifique:

( ) Aprimoramento. Especifique:

( ) Outros. Especifique:

3- Quanto ao trabalho de pesquisa que você desenvolveu durante o curso, ele foi: publicado?

( ) $\mathrm{Sim}$

( ) Não. Por quê?

apresentado em evento científico?

( ) $\mathrm{Sim}$

( ) Não. Por quê?

implementado a sua prática diária?

( ) $\mathrm{Sim}$

( ) Não. Por quê?

4- Você desenvolveu outras pesquisas após a conclusão do curso?

( ) Não

( ) Sim. Quantos?

5- Analise as alternativas abaixo, a contribuição que o curso de especialização em cuidados intensivos lhe proporcionou, seguindo as seguintes opções: Muito (M), Pouco (P) ou Nenhuma (N):

( ) ascensão na carreira

( ) aumento salarial

( ) conhecimento técnico-científico

( ) desenvolvimento de pesquisas

( ) desempenho nas atividades de assistência ao paciente crítico

( ) relacionamento com a equipe multidisciplinar

( ) relacionamento com paciente e família

( ) outras. Especifique:

6- Comentários e sugestões 


\section{REFERÊNCIAS BIBLIOGRÁFICAS}

01. ALMEIDA, M.M.G. de. Pesquisa como instrumento da prática. Rev.Bras.Enfermagem, v.48, n.2, p.155-60, 1995.

02. ANGERAMI, E.L.S. O desenvolvimento da pesquisa no Brasil. Rev.latino-am.enfermagem, v.1, p.43-52, 1993. Número especial.

03. BARRY, P.D. Effects of the critical care unit on the nurse. In: HUDAK, C. M.; GALLO, B.M.; 04. BENZ, J.J. Critical care nursing: a holistic approach. 4.ed. Philadelphia: Linppicott, 1986. Cap. 38, p.661-9.

05. BRASIL. Leis, decretos, etc. Resolução n ${ }^{\circ}$ 14/77. Documenta, Brasília, v.205, p.487-88, 1979.

06. CLARK, S.; FONTAINE, D.K. SIMPSON, T. Recognition, assessment and treatment of anxiety in the critical care setting. Crit.Care Nurse, v.14, n.4, p.2-14, 1994. Supplement.

07. FERNANDES, R.A.Q.; SILVA, S.H. da. Modalidade de fomento à pesquisa na área assistencial. Rev.Bras.Enfermagem, v.48, n.1, p.78-84, 1995.

08. KOIZUMI, M.S. et al. Seleção de candidatos aos cursos de especialização. Rev.Esc.Enfermagem USP, v.19, n. 2, p.111-9, 1985.

09. KOIZUMI, M.S. et al. Análise retrospectiva das pesquisas de enfermagem em terapia intensiva: 1975-1984. Rev.Esc.Enfermagem USP, v.20, n. 1, p.5-17, 1986.
10. PADILHA, K.G. Des-cuidar: as representações sociais dos enfermeiros de UTI sobre as ocorrências iatrogênicas de enfermagem. São Paulo, 1994. 188p. Tese (Doutorado) - Escola de Enfermagem, Universidade de São Paulo.

11. PADILHA, K.G.; KIMURA, M.; MIYADAHIRA, A.M. Produção científica de enfermagem em terapia intensiva no Brasil: análise retrospectiva de 1985 a 1994. São Paulo: EEUSP, 1995./ Mimeografado/

12. SILVA, C.M. da et al. Pesquisa em enfermagem: importância e sua evolução no Brasil. Acta Paul. Enfermagem, v.4, n.1, p.34-8, 1991.

13. SOUSA, M.F. de; GUTIÉRREZ, M.G.R. Pesquisa em enfermagem. Acta Paul.Enfermagem, v.3, n.4, p.137-42, 1990 .

14. SPÍNDOLA, T.; CASTAÑON, F.F.; LOPES, G.T. O estresse na UTI. Amb.Hosp., v.6, n.5, p.2541, 1994.

15. TAKAHASHI, E.I.U. As fontes de estresse emocional que afetam a enfermeira na assistência à criança grave. Rev. Esc. Enfermagem USP, v.19, n.1, p.5-20, 1985.

16. TAKAHASHI, E.I.U. A emoção na prática de enfermagem: relatado por enfermeiros de UTI e UI. São Paulo, 1991. 241p. Tese (Doutorado) Escola de Enfermagem, Universidade de São Paulo.

17. UNIVERSIDADE DE SÃO PAULO. Reitoria. Resolução no 1709/79. Diário Oficial, São Paulo, 16 out 1979 . 